\title{
Internal Avalanches in a Granular Medium
}

\author{
S. S. Manna ${ }^{1 *}$ and D. V. Khakhar ${ }^{2 \dagger}$ \\ ${ }^{1}$ Satyendra Nath Bose National Centre for Basic Sciences, Block-JD, Sector-III, Salt Lake, Calcutta-700091, India \\ ${ }^{2}$ Department of Chemical Engineering, Indian Institute of Technology, Powai, Mumbai - 400076, India \\ *manna@boson.bose.res.in, †khakhar@cupid.che.iitb.ernet.in
}

\begin{abstract}
Avalanches of grain displacements can be generated by creating local voids within the interior of a granular material at rest in a bin. Modeling such a two-dimensional granular system by a collection of mono-disperse discs, the system on repeated perturbations, shows all signatures of Self-Organized Criticality. During the propagation of avalanches the competition among grains creates arches and in the critical state a distribution of arches of different sizes is obtained. Using a cellular automata model we demonstrate that the existence of arches determines the universal behaviour of the model system.
\end{abstract}

PACS numbers: 05.70.Jk, $64.60 \mathrm{Lx}, 74.80 . \mathrm{Bj}, 46.10 .+\mathrm{z}$

The search for self organized criticality (SOC) [1] in granular systems in general and in sandpiles in particular has been a subject of active research over the last decade. How such a system reacts in the form of fast cascades of grain displacements, called 'avalanches', in response to a slow external drive at the microscopic level is the crucial question of study. It has been suggested that starting from an arbitrary initial condition, a non-equilibrium critical state, characterised by scale free avalanches in both space and time, should be expected after a long time $[1,2]$. Other naturally occuring physical phenomena like forest fires [3], river networks [4], earthquakes [5] etc. have also been proposed as examples of systems showing SOC.

It was observed that a steady shaped sandpile grown on a fixed base fulfills all the requirements of SOC. In this state, dropping even a few grains creates rapidly moving avalanches of sand sliding along the surface of the pile. It was expected that the avalanches should be power law distributed in their spatial as well as temporal extents and therefore a sandpile should be considered as a simple example showing SOC $[1,2]$.

Experimental observations, however, show partial support to this idea. Sand is allowed to fall from a slowly rotating semi-cylindrical drum through the space between the plates of a vertical parallel plate capacitor. The Fourier spectrum of the time series data of fluctuating capacitance showed a peak contrary to the expectation of a power law [6]. Similarly sand dropping from the edge of a sandpile on a fixed base was directly measured, and was seen to have a power law distribution only for small systems but not for large systems [7]. It was argued that due to the approximately spherical shape of the grains used in these experiments the effect of inertia cannot be neglected and this was the reason for absence of scaling behaviour. This is verified in an experiment using rice grains, which are highly anisotropic, and criticality was observed [8].

A number of theoretical models, generally known as 'sandpile' models have been studied. The models are based on stochastically driven cellular automata which evolve under a nonlinear, diffusive mechanism leading to a nonequilibrium critical state. The most widely studied is the Abelian sandpile model (ASM) where the stable configuration does not depend on the sequence of sand grain additions [9]. Other variants of the sandpile model include situations where the stability of a sand column depends on the local slope or the Laplacian of the height profile [10]. A two-state sandpile model with stochastic evolution rules is also studied [11].

In all the studies discussed above the avalanches propagate on the surfaces of the sandpiles. However, there exists the possibility of creating avalanches in the interior of a granular material. In a granular material kept in a bin at rest, different grains support one another by mutually acting balanced forces. Now if a grain is removed, the grains which were supported by it become unstable and tend to move. Eventually the grains in the further neighbourhood also loose their stability. As a result an avalanche of grain displacements takes place, which stops when no more grains remain unstable. The basic physical behavior here is thus quite different from the avalanches on the surface of the pile because of the constraints to particle motion in the dense particle beds.

A two-dimensional semi-lattice model was studied for this problem [12]. Non-overlaping unit square boxes model the grains, whose horizontal coordinates can vary continuously where as the vertical co-ordinates are discretized. A grain can only fall vertically if insufficiently supported and sufficient space below is available. The system is disturbed by removing grains at the bottom and thus creating avalanches of grain movements.

During the propagation of avalanches inside a granular medium grains compete locally with one another to occupy the same vacant space. The high packing densities of the particle beds prevent a single particle from occupying the available void space, and consequently particles get locked to form 'arches' [13]. A stable arch is a chain 
of grains where the weight of each grain is balanced by the reaction forces from two neighbouring grains in the chain. Arches can form only when a grain is allowed to roll over other grains. Since the rolling motion of the grains was absent therefore the arches were not formed in the granular patterns [12].

We here study a more realistic model of this problem in two dimension, where both fall and roll motions of grains are allowed. The granular system is represented by $N$ hard monodisperse discs of radii $R$. No two grains are allowed to overlap but can touch each other and one can roll over the other if possible without slipping. A rectangular area of size $L \times L$ on the $x-y$ plane represents our two dimensional bin. Periodic boundary condition is used along the $x$ direction and the gravity acts along the $-y$ direction. The bottom of the bin at $y=0$ is highly sticky and any grain which comes in contact gets stuck there and does not move further.

The dynamical evolution of the system is studied by a 'pseudo-dynamics' [14]. Unlike the method of molecular dynamics we donot solve here the classical equations of motion for the grain system. Only the direction of gravity and the local geometrical constraints due to the presence of other grains govern the movement of a grain. For justification of using the pseudo-dynamics we argue that due to the high compactness of the system a grain never gets sufficient time to accelerate much. Therefore in our model, a grain can have only two types of movements in unit time: The vertical fall up to a maximum distance $\delta$ and the roll up to a maximum angle $\theta=\delta / 2 R$ over another grain in contact.

Movement of a grain needs the information on the positions of other grains in the neighbourhood which it may possibly interact. An efficient way to search this is to digitize the bin into a square grid and associate the serial number of a grain to the primitive cell of the grid containing its centre. Choise of $R=1 / \sqrt{2}+$ ensures that a cell corresponds to at most one grain. With this choice it is sufficient to search only within the 24 neighbouring cells for possible contact grains. The weight of a grain $n$ is supported by two other grains. If $n_{L}$ and $n_{R}$ are the serial numbers of the left $(L)$ and right $(R)$ supporting grains, then the grain $n$ is updated as: (i) If $n_{L}=n_{R}=0$, it falls, (ii) if $n_{L} \neq 0$ but $n_{R}=0$, it rolls over $n_{L}$, (iii) if $n_{L}=0$ but $n_{R} \neq 0$, it rolls over $n_{R}$, (iv) if $n_{L} \neq 0$ and $n_{R} \neq 0$ it is stable.

When the grain $n$ with the centre at $\left(x_{n}, y_{n}\right)$ is allowed to fall, it may come in contact with a grain $r$ at $\left(x_{r}, y_{r}\right)$ within the distance $\delta$. The new coordinates are:

$$
\begin{gathered}
y_{n}^{\prime}=y_{r}+\sqrt{4 R^{2}-\left(x_{n}-x_{r}\right)^{2}} \text { if } y_{n}-y_{n}^{\prime}<\delta \\
\text { otherwise, } y_{n}^{\prime}=y_{n}-\delta .
\end{gathered}
$$

Similarly the grain $n$, while rolling over the grain $r$, may come in contact with another grain $t$ at $\left(x_{t}, y_{t}\right)$. The new coordinates for the centre of $n$ where it touches both $r$ and $t$ are:

$$
\begin{aligned}
& x_{n}^{\prime}=\frac{1}{2}\left(x_{t}+x_{r}\right)+g\left(y_{t}-y_{r}\right) \sqrt{\frac{4 R^{2}}{d_{r t}^{2}}-\frac{1}{4}} \\
& y_{n}^{\prime}=\frac{1}{2}\left(y_{t}+y_{r}\right)-g\left(x_{t}-x_{r}\right) \sqrt{\frac{4 R^{2}}{d_{r t}^{2}}-\frac{1}{4}} .
\end{aligned}
$$

Here $d_{r t}$ is the distance between the centres of the grains $r$ and $t$ and the $g=+1$ and -1 for left and right rolls. To reach the new position if the grain $n$ rolls an angle $\theta_{m}<\theta$ then it is accepted, otherwise it rolls up to an angle $\theta$.

The initial grain pattern is generated by the ballistic deposition method with restructuring $[15,16]$. Grains are released sequentially one after another along randomly positioned vertical trajectories and are allowed to fall till they touch the growing pile and then roll down along the paths of steepest descent to their local stable positions. We notice that in the initial pattern no big arch exists since a grain while rolling down along the surface does not need to compete with any other grain. Using a system of $L=80$, we compute the packing fraction $\rho=0.822 \pm$ 0.005 consistent with more precise estimate of $0.8180 \pm$ 0.0002 in [16].

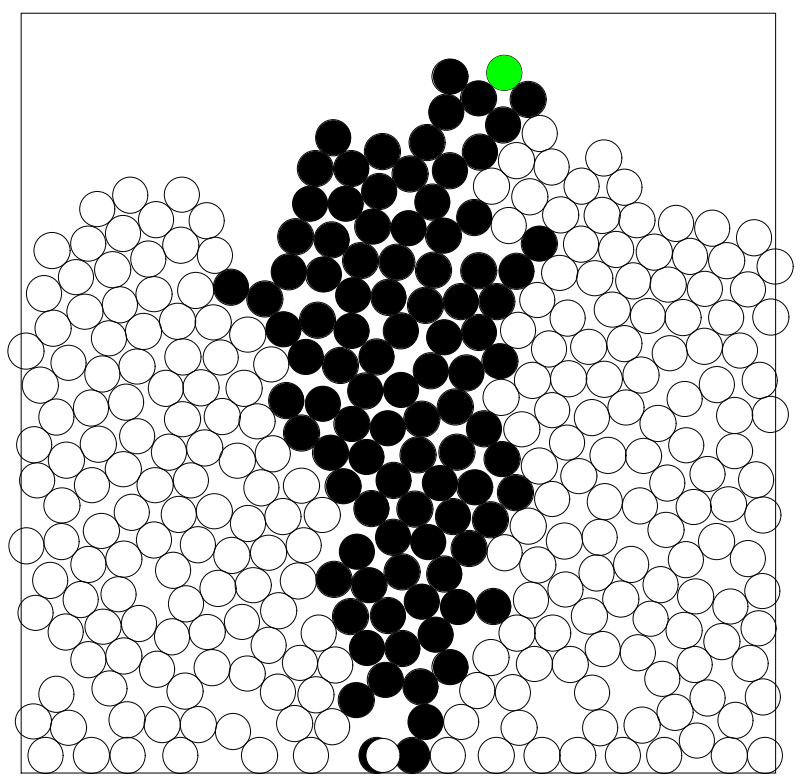

FIG. 1. Picture of a typical avalanche. The vacant circles denote the undisturbed grains, filled circles denote the displaced grains, the opaque circle at the bottom denotes the position of the grain which was removed and the shaded circle denote the position of the grain where the removed grain was replaced. In a system of size $L=30$ and number of grains $N=340,97$ grains took part in the avalanche.

The system is repeatedly perturbed by removing grains randomly at the bottom one after the other. Every time 
a grain is removed, an avalanche is followed and after the avalanche is over the removed grain is placed back at a random position on the top surface again using the same ballistic method (Fig. 1). We first observe that $\rho$ of the stable configuration, averaged over many initial random patterns generated with the same algorithm, decreases with the number of avalanches created and finally reaches a steady value of $0.748 \pm 0.005$. A similar study but with different initial configurations with a different value of average initial $\rho$, shows the final steady state packing fraction reaches the same value, which implies that the final stable state is independent of the initial state. The total number of grain displacements is called the avalanche size $s$ and the duration of the avalanche is the life time $t$. Power law distributions are observed for $s$ and $t: D(s) \sim s^{-\tau_{s}}$ and $D(t) \sim t^{-\tau_{t}}$ with $\tau_{s} \approx 1.7$ (Fig. 2) and $\tau_{t} \approx 2.1$.

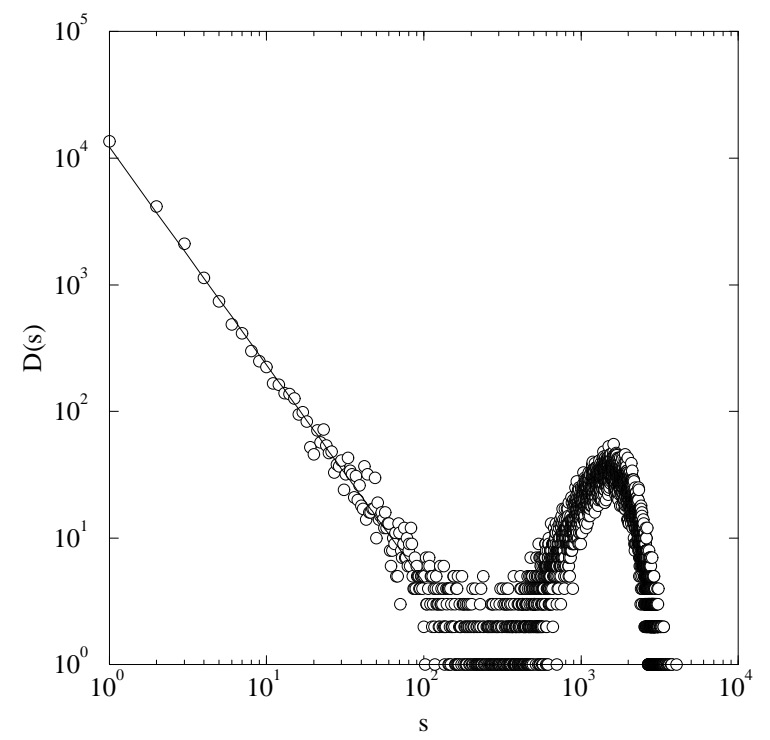

FIG. 2. Plot of the avalanche size distribution $D(s)$ vs. $s$ for a system of size $N=10000$ and over 70000 avalanches. The straight part fits with a slope $\tau_{s}=1.7$.

The observed exponents in this model are significantly larger than the corresponding values of $\tau_{s} \approx 1.34$ and $\tau_{L} \approx 1.47 \mathrm{in} \mathrm{[12].} \mathrm{We} \mathrm{argue} \mathrm{that} \mathrm{the} \mathrm{reason} \mathrm{for} \mathrm{this}$ difference could be the existence of arches in our model. Due to the arches, the propagation of avalanches get arrested more frequently than in [12] and therefore smaller avalanches are more probable which enhances the values of the critical exponents in our model.

To demonstrate more explicitly that the above reasoning may be correct, we study a cellular automata model for the granular systems. A square lattice of size $L$ with periodic boundary condition along the $x$ axis represents the bin. The gravity acts in the $-y$ direction. Positions of the grains are limited only to the lattice sites: a site can be either occupied by a grain or remain vacant. Initially the bin is filled up again using the ballistic method.

A single movement of a grain at $C(i, j)$ involves the neighbouring seven sites: $L U(i-1, j+1), L(i-$ $1, j), L D(i-1, j-1), D(i, j-1), R D(i+1, j-1), R(i+1, j)$ and $R U(i+1, j+1)$.In the fall move the grain comes down one level to the vacant site at $D$ and in roll move the grain goes either to the vacant site at $L D$ or at $R D$ (Fig. 3). An arch is formed when a grain at $C$ is considered stable if both of its two diagonally opposite sites either at $L D$ and $R U$ or, at $L U$ and $R D$ are occupied. As a result, on the square lattice, the only possible shape of an arch consists of two sides of a triangle. Depending on whether we allow the arch formations or not, we define the following two models.

fall
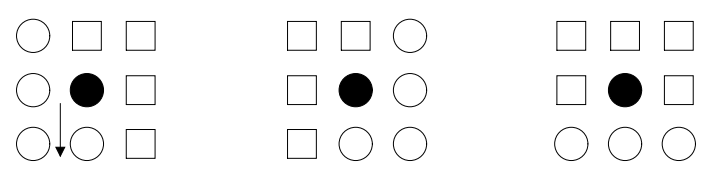

roll
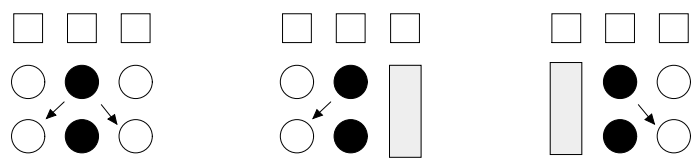

FIG. 3. The possible fall and roll moves in the cellular automata model of the granular system on the square lattice. Filled circle denotes the position of a grain, unfilled circle denotes a vacant site. The grain moves to the vacant position irrespective of the occupation of the sites with square boxes. Shaded rectangle denotes a pair of sites, in which at least one is occupied.

In model A we allow arch formations. The grain at $C$ falls only if any of the following three conditions is satisfied: (i) $L D, L$ and $L U$ are vacant (ii) $R D, R$ and $R U$ are vacant (iii) $L D$ and $R D$ are vacant. In all other situations the grain does not fall. Notice that in conditions (i) and (ii) we are allowing the formation of arches. The grain at $C$ rolls only if the site $D$ is occupied. This is done in any of the three following ways: (i) If both $L D, L$ are vacant but either of $R D, R$ is occupied then the grain rolls to $L D$. (ii) if both $R D, R$ are vacant but either of $L D, L$ is occupied then the grain rolls to $R D$. (iii) If all four sites at $L D, L, R D, R$ are vacant then the grain rolls either to $L D$ or to $R D$ with probability $1 / 2$. A steady state pattern is shown in Fig. 4.

In model $\mathrm{B}$ we do not allow arch formations. The first two conditions for fall of model $A$ are modified as: (i) $L D$ and $L$ are vacant (ii) $R D$ and $R$ are vacant. All other conditions of fall as well as roll remain same as in model A. 
Initial granular patterns are generated using the same random ballistic deposition method with restructuring $[15,16]$ and patterns are same for both models. As before the avalanches are created by taking out one grain at a time at the bottom, allowing the system to relax and replacing it randomly on the surface after the avalanche is over. Here also we see that the average density of sites, starting from an initial value of $0.907 \pm 0.005$, decreases to the final stable value of $0.590 \pm 0.005$ in model $\mathrm{A}$ and to $0.618 \pm 0.005$ in model B. The avalnche size $s$ and life time $t$ follow power law distributions: $D(s) \sim s^{-\tau_{s}^{A}}, D(t) \sim$ $t^{-\tau_{t}^{A}}$ and similarly for the model B. Different exponents are obtained for the two models: $\tau_{s}^{A} \approx 1.48$ and $\tau_{t}^{A} \approx$ 1.99 where as $\tau_{s}^{B} \approx 1.34$ and $\tau_{t}^{B} \approx 1.50$. We explain that absence of arches makes the exponent values for the model B close to that of [12] but their presence enhances the values in model $\mathrm{A}$.

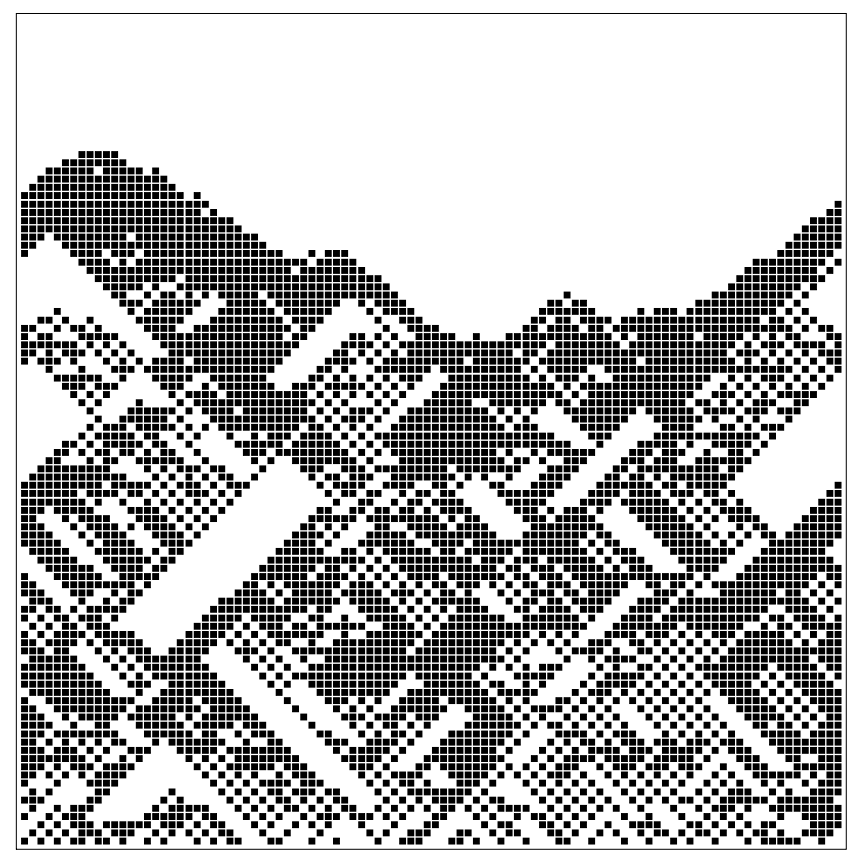

FIG. 4. Figure 4: A stable configuration of the granular system in the cellular automata model A after a large number of avalanches are created. Triangular arches are noticed.

To summarise, avalanches of grain displacements can be created in the interior of a granular material at rest by locally disturbing the system. In a numerical study we provide indications that on repeated creations of such avalanches, the granular system reaches a critical state characterised by long range correlations. The presence of arches play an important role in determining the critical behaviour. Since in an avalanche grain motions are highly constrained, the effect of inertia may not be very significant. Therefore, we conjecture that in real experiment of internal avalnches it should be possible to observe SOC even with spherical grains unlike the case of surface avalanches, where anisotropic grains were necessary to observe criticality.

The funding provided by the Indo-French Centre for the Promotion of Advanced Research (IFCPAR) is gratefully acknowledged.

[1 ] P. Bak, C. Tang and K. Wiesenfeld, Phys. Rev. Lett. 59. 381 (1987).

[2 ] P. Bak, C. Tang and K. Wiesenfeld, Phys. Rev. A 38, 364 (1988); P. Bak, How Nature Works: The Science of Self-Organized Criticality, (Copernicus, New York, 1996).

[3 ] P. Bak and K. Chen, Physica D 38, 5 (1989), HM Bröker and P. Grassberger, Phys. Rev. E 56, R4918 (1997).

[4 ] A. Rinaldo, I. Rodriguez-Iturbe, R. Rigon, E. Ijjasz-Vasquez and R. L. Bras, Phys. Rev. Lett. 70, 822 (1993); S. S. Manna and B. Subramanian, Phys. Rev. Lett. 76 (1996) 3460.

[5 ] J. M. Carlson and J. S. Langer, Phys. Rev. Lett. 62, 2632 (1989); Z. Olami, H. J. S. Feder and K. Christensen, Phys. Rev. Lett. 68, 1244 (1992).

[6 ] H. M. Jaeger, C-h Liu and S. R. Nagel, Phys. Rev. Lett. 62, 40 (1989).

[7 ] G. A. Held, D. H. Solina II, D. T. Keane, W. J. Haag, P. M. Horn and G. Grinstein, Phys. Rev. Lett. 65, 1120 (1990).

[8 ] V. Frette, K. Christensen, A. Malthe-Sorenssen, J. Feder, T. Jossang and P. Meakin, Nature (London) 379, 49 (1996).

[9 ] D. Dhar, Phys. Rev. Lett. 64, 1613 (1990).

[10 ] L. P. Kadanoff, S. R. Nagel, L. Wu and S. Zhou, Phys. Rev. A. 39, 6524 (1989); S. S. Manna, Physica A 179, 249 (1991).

[11 ] S. S. Manna, J. Phys. A 24, L363 (1992).

[12 ] R. E. Snyder and R. C. Ball, Phys. Rev. E 49, 104 (1994).

[13 ] D. E. Wolf, in Computational Physics ed. by K. H. Hoffmann and M. Schreiber, Springer 1996.

[14 ] S. S. Manna and D. V. Khakhar, in Nonlinear Phenomena in Material Science III, G. Ananthakrishna, L. P. Kubin and G. Martin (Eds.) (Transtech, Switzerland, 1995).

[15 ] W. M. Visser and M. Bolsterli, Nature 239, 504 (1972).

[16 ] R. Jullien, P. Meakin and A. Pavlovitch, in Disorder and Granular Media, D. Bideu and A. Hansen (Eds.) (Elsevier 1993). 